\title{
Completing the RANZCP scholarly project in a rural/regional setting: a practical example
}

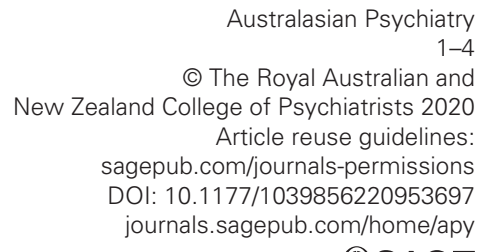

@SAGE

\author{
Harry Hill ID Mental Health Service, South West Healthcare, Australia; and Deakin University, Australia \\ Carol Harvey Department of Psychiatry, The University of Melbourne, Australia; and North West Area Mental Health Service, \\ North Western Mental Health, Australia
}

\begin{abstract}
Objective: The Royal Australian and New Zealand College of Psychiatrists (RANZCP) scholarly project (SP) is a major hurdle for trainees completing psychiatric training - intensified for those working in rural settings. Whilst strategies to overcome this challenge have been proposed, there are few examples of how these have been successfully implemented. This report will describe the completion of a RANZCP SP in a regional setting, with supervision and support provided by an academic psychiatrist working within a metropolitan research centre.

Conclusion: Trainees in remote geographical locations can utilise expert supervision from academic psychiatrists to successfully complete the SP. Key components are support from both the trainee and supervisor's employers, utilisation of a range of supervision modalities and a clear pragmatic memorandum of understanding between the supervisor and trainee. This initiative had widespread benefits beyond completion of the SP for all parties involved.
\end{abstract}

Keywords: scholarly project, regional, supervision, training, psychiatry

$\mathrm{T}$ he Royal Australian and New Zealand College of Psychiatrists (RANZCP) scholarly project (SP) is a mandatory component of psychiatry training in Australia and New Zealand, strengthening a culture of research and academic psychiatry. ${ }^{1}$ Aligning with the regional psychiatric zeitgeist, the SP focuses on the CanMEDS[AQ: 1] framework of research and the promotion of evidence-based practice. ${ }^{1,2}$ Despite being a core component of training, trainees have reported difficulties in completing this assessment, identifying lack of protected time for research, access to appropriate supervision and the numerous other college requirements as key factors in delaying SP completion. ${ }^{2}$

These difficulties are magnified in regional Australia, where there is a severe shortage of psychiatrists, ${ }^{3}$ further exacerbated by a lack of psychiatrists with experience in supervising trainees in completing the SP or with significant academic psychiatric experience. ${ }^{1}$ Qualitative research has identified the struggles that rural trainees report in sourcing appropriate SP supervisors. ${ }^{1}$ Rural trainees report feeling disadvantaged due to limited peer contact, difficulty in accessing formal aspects of training and feeling educationally isolated. ${ }^{4}$ The experience and ease of completing the SP has been described as likely to be highly influenced by the supervision received. ${ }^{5}$
Whilst previous studies have described potential strategies to overcome challenges in completing the SP, 1,5 there are few examples of how these have been successfully implemented by trainees. This report will describe a practical, real-life example of completing the RANZCP SP in a rural setting, with supervision and support provided by an academic psychiatrist working within a metropolitan research centre.

\section{Origins}

The experience commenced pragmatically, following the trainee's desire to explore research opportunities in psychiatric rehabilitation. Working in a rural setting, access to an experienced supervisor with an interest in this area was limited. The trainee identified an expert supervisor with a strong interest in psychiatric rehabilitation during a formal education program, and fostered this relationship through a meeting at the RANZCP congress. The

Corresponding author:

Harry Hill, Mental Health Service, South West Healthcare, Warrnambool, VIC 3280, Australia.

Email: hphill88@gmail.com 
academic psychiatrist then proposed that they meet to explore SP possibilities.

\section{Logistics}

With the support of the regional employer, the trainee met with the potential supervisor to assess supervision opportunities and potential scholarly endeavours. Three well-defined projects, suitable for a SP, were identified that were aligned with the trainee's interests and supervisor's academic pursuits. With the supervisor's support, the trainee explored the academic merit and suitability of each project. Having identified a project, the trainee and supervisor worked on a pragmatic memorandum of understanding regarding the trainee/supervisor arrangement, which was in turn supported by both the trainee's and the metropolitan-employed supervisor's organisations.

\section{Supervision}

Supervision was provided over an 18-month period across multiple modalities - combining both face-to-face, videoconferencing, telephone and email correspondence. Freely available software-based videoconferencing solutions were utilised and reliable internet was available at both sites. The trainee and supervisor met regularly throughout the project. In line with previous guidance, ${ }^{5}$ most aspects of the project could be completed without face-to-face supervision.

From the trainee's perspective, the supervisor provided a caring attitude, delivering constructive feedback, keeping the trainee accountable and ensuring achievable timelines. ${ }^{6}$ The supervisor guided the trainee through exploration of relevant literature, hypothesis refinement, data collection and analysis as well as completing project write-up. ${ }^{6}$

\section{Becoming part of a research community}

Whilst rural trainees often report educational isolation, in this example the trainee became part of a team - inadvertently, becoming part of a research community. Through engagement in the project, the trainee was able to work within the supervisor's research unit, drawing on the assistance and expertise of the unit's research assistant as well as other academics. Through this collaboration, the trainee was able to access the Victorian Mental Health, Health Sciences Library, allowing access to the library's extensive electronic database and resources.

Given the preliminary work previously undertaken to plan the project, ${ }^{7}$ the trainee collaborated with an international group of experts in psychiatric rehabilitation across the United Kingdom, Hong Kong, India and Australia. Without the networks already established by the supervisor, this scope and reach would clearly not have been possible.

\section{Achievements: publication and presentation}

Medical students and junior doctors are often concerned about completing rural training due to concerns around academic opportunity and career progression. ${ }^{8,9}$ Highlighting achievements obtained in rural and regional placements is important in addressing these fears.

The supervisor and expert group ${ }^{7}$ were crucial in navigating the project through the publication process, assisting the trainee to feel comfortable in completing a novel process. ${ }^{7}$

The supervisor and one of the international experts encouraged the trainee to apply for an award offered by the Royal College of Psychiatrists (RCPsych), UK. The project was judged to be of sufficient academic merit to be awarded the Douglas Bennett prize (RCPsych), ${ }^{10}$ resulting in an opportunity for the trainee to present the project internationally (RCPsych $)^{10}$ and later, locally, at the Section of Social, Cultural and Rehabilitation Psychiatry conference. ${ }^{11}$

\section{Positive secondary outcomes}

There were numerous positive secondary outcomes that were not anticipated when commencing the SP arrangement.

As a result of presenting at the local Section conference and due to the expansion of the Section to include rehabilitation, ${ }^{11}$ the trainee was invited to - and accepted - a role within the Section as a co-opted committee member.

The trainee's interest in rehabilitation psychiatry was enhanced, and ultimately, the trainee was able to disseminate their learnings within their rural service. One prime example was the trainee's ability to assist in the facilitation of a multifamily group for people with psychosis - a first for the service.

From the trainee perspective, whilst not initially expected, but definitely greatly appreciated, was the guidance, support and mentorship the supervisor provided outside of the realm of the SP; helping the trainee to navigate RANZCP examinations, career progression and balancing work-life demands.

There were benefits for the supervisor, who was able to complete and publish a planned project in a timely fashion, which had not progressed due to lack of available time and resources. The SP provided the supervisor with the satisfaction associated with mentoring and supporting a junior psychiatrist. Other supervisor benefits included: enhanced knowledge of rural training experiences and the opportunity to build upon the trainee's 
enthusiasm and interest in rehabilitation to build capacity within the RANZCP.

\section{Barriers and challenges}

Perhaps surprisingly, there were no major barriers identified in establishing supervision or in the SP completion.

Whilst it was hypothesised that having the trainee and the supervisor working in different organisations, large distances apart, could be a barrier, this was overcome and largely negated through the use of technology and the support of both the trainee and the supervisor's employers; the trainee's service was appreciative of the trainee's exposure to, and supervision from, an expert in their area of interest, and the supervisor's employer viewed the initiative as part of their core business in supporting trainee academic endeavour. A key component was making this an enjoyable and mutually beneficial arrangement for all involved, ensuring a clear framework around supervisor and trainee expectations, and assurance of accountability. Central to this occurring was having an experienced supervisor, as well as a trainee who was willing to take the initiative in seeking appropriate support and guidance.

\section{Conclusion}

This description is the first real-world example detailing how the challenges involved in completing the SP by a rural/regional trainee, including lack of access to appropriate supervision and educational isolation, can be overcome. This initiative demonstrates that it is possible to complete the SP as a rural trainee; it shows that the establishment of supervision arrangements for rural trainees with major research centres and academic psychiatrists is achievable, with widespread benefits, such as becoming part of a research community. Whilst this example describes the experience of a rural trainee, it is not unreasonable that this model could also be applied to metropolitan trainees who don't have access to appropriate supervisors within their workplace.

Support from both the trainee and the supervisor's employers was crucial to guaranteeing the viability of this arrangement. A fundamental factor was ensuring that the project was mutually beneficial for all parties involved. Techniques, such as better access to, and use of, online resources such as video-conferencing and remote library access, should be evaluated in relation to successful completion of the scholarly project, as they have been in clinical applications. ${ }^{12}$

We hypothesise that this situation is not unique, that there are potentially numerous trainees wishing to complete projects in their areas of interest without access to relevant academic expertise in their employing service. Equally, there are likely many academic psychiatrists with ready-made projects, looking for energetic and motivated trainees to undertake much needed work of academic merit. ${ }^{5}$ We question if trainees are aware of the possibility of seeking external supervision, or if they lack confidence that potential approaches for supervision will be well received? It is hoped that this description can not only abate any concerns these trainees may have, but also highlight the potential shared benefits of endeavours, such as those described.

Whilst the project required dedication and commitment from the trainee to achieve a desired outcome, the relative simplicity of processes involved to overcome previously described challenges should provide a sense of hope, confidence and motivation that successful completion of the SP is achievable. We recognise that this project relied on considerable goodwill, which may not be universally transferable. Future research should assess how translatable the approaches described are when applied to a range of services, supervisors and trainees.

Training and retaining a rural workforce is a significant concern for the RANZCP and medical community.3,9 Future initiatives should look at ways of better connecting and supporting trainees and supervisors with shared research interests, perhaps through establishing databases of available supervisors and projects. Rural services should be proactive in supporting their trainees to complete projects with local supervisors, should they be available, but they should also promote collaboration with research centres of excellence.

\section{Acknowledgements}

The authors would like to thank Catherine Bressanutti, Helen Killaspy, Padmavati Ramachandran, Roger Man Kin Ng and Nicole Bulman for their expertise and contribution to the scholarly project described.

\section{Disclosure}

The authors report no conflict of interest. The authors alone are responsible for the content and writing of the paper.

\section{Funding}

The authors received no financial support for the research, authorship, and/or publication of this article.

ORCID iD

Harry Hill iD https://orcid.org/0000-0002-8239-3228

\section{References}

1. Ng F, Ayres A, Suetani S, et al. Stepping forward: challenges and pathways to building a vibrant research culture through the scholarly project. Australas Psychiatry 2019; 27: 187-191.

2. Cheung $\mathrm{G}$, Gale $\mathrm{C}$ and Menkes $\mathrm{DB}$. What affects completion of the scholarly project? $\mathrm{A}$ survey of RANZCP trainees. Australas Psychiatry 2018; 26: 545-555.

3. Department of Health. Australia's Future Health Workforce - Psychiatry, https://www. health.gov.au/internet/main/publishing.nst/Content/597F2D320AF16FDBCA257F7C008 0667F/\$File/AFHW\%20Psychiatry\%20Report.pdf (2016, accessed 26 November 2019).

4. Nash L, Hickie C, Clark S, et al. The experience of psychiatry training in rural NSW. Australas Psychiatry 2014; 22: 492-499.

5. Suetani S, Gill S and Galletly C. A scholarly endeavour: some practical tips on completing the scholarly project. Australas Psychiatry 2015; 23: 29-31.

6. Cheung G, Friedman SH, Ng L, et al. Supervising trainees in research: what does it take to be a scholarly project supervisor? Australas Psychiatry 2018; 26: 210-213. 
7. Hill H, Killaspy H, Ramachandran P, et al. A structured review of psychiatric rehabilitation for individuals living with severe mental illness within three regions of the Asia-Pacific: implications for practice and policy. Asia-Pac Psychiatry 2019; 11: e12349.

8. Jones G, DeWitt D and Cross M. Medical students' perceptions of barriers to training at a rural clinical school. Rural Remote Health 2007; 7: 685.

9. Cuesta-Briand B, Coleman M, Ledingham R, et al. Understanding the factors influencing junior doctors' career decision-making to address rural workforce issues: testing a conceptual framework. Int J Environ Res Public Health 2020; 17: 537.
10. Royal College of Psychiatrists. Prizes and bursaries, https://www.rcpsych.ac.uk/members/ your-faculties/rehabilitation-and-social-psychiatry/prizes-and-bursaries (2019, accessed 26 November 2019).

11. The Royal Australia and New Zealand College of Psychiatrists. Section of social, cultural and rehabilitation psychiatry, https://www.ranzcp.org/membership/faculties-sectionsand-networks/sscrp (accessed 26 November 2019).

12. García-Lizana F and Muñoz-Mayorga I. What about telepsychiatry? A systematic review. Prim Care Companion J Clin Psychiatry 2010; 12: PCC.09m00831. 


\section{University Library}

\section{- M M I N E R VA A gateway to Melbourne's research publications}

Minerva Access is the Institutional Repository of The University of Melbourne

Author/s:

Hill, H;Harvey, C

Title:

Completing the RANZCP scholarly project in a rural/regional setting: a practical example

Date:

2021-07-01

Citation:

Hill, H. \& Harvey, C. (2021). Completing the RANZCP scholarly project in a rural/regional setting: a practical example. Australasian Psychiatry, 29 (2), pp.234-236. https:// doi.org/10.1177/1039856220953697.

Persistent Link:

http://hdl.handle.net/11343/274709 